\title{
Genetic susceptibility to hip arthroplasty failure-association with the RANK/OPG pathway
}

\author{
Mohammad Malik
}

Published online: 15 November 2007

(C) Springer-Verlag 2007

It has belatedly come to the authors' attention that an inadvertent error in data manipulation has led to an erroneous association between OPD-163 and hip arthroplasty failure.

The raw data have now been re-examined and Table 1 now should read as follows:

The previously specified association between RANK +575 and aseptic failure remains unchanged.

The authors apologise for this error.

The online version of the original article can be found at http://dx.doi. org/10.1007/s00264-006-0074-2.

M. Malik $(\square)$

University of Manchester, CIGMR,

Stopford Building, Oxforg Road,

Manchester M13 9PT, UK

e-mail: hammymalik@hotmail.com
Table 1 SNP allele and genotype frequencies in controls, aseptic and septic THA failures (numbers in parentheses)

\begin{tabular}{|c|c|c|c|}
\hline SNP & Control & Aseptic & Septic \\
\hline \multicolumn{4}{|l|}{ OPG-163 } \\
\hline Number of subjects & 150 & 91 & 63 \\
\hline \multicolumn{4}{|l|}{ Allele frequency } \\
\hline A & $0.83(249)$ & $0.87(158)$ & $0.86(108)$ \\
\hline G & $0.17(51)$ & $0.13(24)$ & $0.14(18)$ \\
\hline \multicolumn{4}{|l|}{ Genotype frequency } \\
\hline $\mathrm{A} / \mathrm{A}$ & $0.71(106)$ & $0.76(69)$ & $0.73(46)$ \\
\hline $\mathrm{A} / \mathrm{G}$ & $0.25(38)$ & $0.22(20)$ & $0.25(16)$ \\
\hline $\mathrm{G} / \mathrm{G}$ & $0.04(6)$ & $0.02(2)$ & $0.02(1)$ \\
\hline \multicolumn{4}{|l|}{ OPG-245 } \\
\hline Number of subjects & 149 & 91 & 62 \\
\hline \multicolumn{4}{|l|}{ Allele frequency } \\
\hline $\mathrm{T}$ & $0.91(270)$ & $0.92(167)$ & $0.95(118)$ \\
\hline G & $0.09(28)$ & $0.08(15)$ & $0.05(6)$ \\
\hline \multicolumn{4}{|l|}{ Genotype frequency } \\
\hline $\mathrm{T} / \mathrm{T}$ & $0.81(121)$ & $0.84(76)$ & $0.90(56)$ \\
\hline $\mathrm{T} / \mathrm{G}$ & $0.19(28)$ & $0.16(15)$ & $0.10(6)$ \\
\hline $\mathrm{G} / \mathrm{G}$ & 0 & 0 & 0 \\
\hline \multicolumn{4}{|l|}{ OPG+1181 } \\
\hline Number of subjects & 147 & 89 & 62 \\
\hline \multicolumn{4}{|l|}{ Allele frequency } \\
\hline $\mathrm{C}$ & $0.56(166)$ & $0.54(96)$ & $0.57(71)$ \\
\hline $\mathrm{G}$ & $0.44(128)$ & $0.46(82)$ & $0.43(53)$ \\
\hline \multicolumn{4}{|l|}{ Genotype frequency } \\
\hline $\mathrm{C} / \mathrm{C}$ & $0.29(43)$ & $0.31(28)$ & $0.39(24)$ \\
\hline $\mathrm{C} / \mathrm{G}$ & $0.54(80)$ & $0.45(40)$ & $0.37(23)$ \\
\hline $\mathrm{G} / \mathrm{G}$ & $0.17(24)$ & $0.24(21)$ & $0.24(15)$ \\
\hline \multicolumn{4}{|l|}{ RANK+575 } \\
\hline Number of subjects & 144 & 85 & 62 \\
\hline \multicolumn{4}{|l|}{ Allele frequency } \\
\hline $\mathrm{C}$ & $0.82(154)$ & $0.39(67)$ & $0.57(71)$ \\
\hline $\mathrm{T}$ & $0.18(134)$ & $0.61(103)$ & $0.43(53)$ \\
\hline \multicolumn{4}{|l|}{ Genotype frequency } \\
\hline $\mathrm{C} / \mathrm{C}$ & $0.31(44)$ & $0.13(11)$ & $0.35(22)$ \\
\hline $\mathrm{C} / \mathrm{T}$ & $0.46(66)$ & $0.53(45)$ & $0.44(27)$ \\
\hline $\mathrm{T} / \mathrm{T}$ & $0.23(34)$ & $0.34(29)$ & $0.21(13)$ \\
\hline
\end{tabular}

